\title{
Detection in liquid chromatography based on different luminescence principles
}

\author{
R.W. Frei, N.H. Velthorst and C. Gooijer \\ Department of General and Analytical Chemistry, Free University, \\ De Boelelaan 1083, 1081 HV Amsterdam, The Netherlands
}

\begin{abstract}
Various luminescence techniques based on the use of intermolecular energy transfer phenomena for detection in liquid streams are discussed. The two distinct methods based on room temperature phosphorescence in liquid phases (RTPL), the sensitized and quenched RTPL, are described. In particular the potential of phosphorescence quenching is wide. It will be demonstrated that this method has considerable promise for the determination of organic compounds, but also as a detector for selected inorganic ions, e.g., $\mathrm{NO}_{2}^{-}, \mathrm{SO}_{3}{ }^{2-}, \mathrm{Pt}^{2+}$ in ion chromatography. Furtheron attention is paid to photochemically generated or photosensitized reactions. It is shown that photochenical reactions can be used as a special type of post-column derivatization, and inprovements in both sensitivity and selectivity have been demonstrated. The mechanisms for photoreduction of anthraquinone by isopropanol and for the sensitized photo-oxygenation of ethanol are discussed.
\end{abstract}

\section{INTRODUCTION}

Luminescence techniques have become increasingly popular for detection in liquid chromatography. Owing to their inherent sensitivity and selectivity conventional fluorescence has been the most important and most generally used principle and many pre- and post-column chemical derivatization techniques have been developed to render non- or poorly fluorescent compounds suitable to this detection mode (Ref. 1). Besides classical fluorescence spectroscopy some other principles have gradually emerged in the recent past such as laser excited luminescence, cheniluninescence, use of solid state (catalytic) reactors to induce luminescence, photosensitized luminescence and also various modes of phosphorescence spectroscopic techniques. Some of these newer techniques shall be discussed in the following sections and some trends for future developments shall be suggested.

\section{ROOM TEMPERATURE PHOSPHORESCENCE IN LIQUIDS (RTPL)}

The phosphorescence principle similar to fluorescence is best visualized by using a simplified Jablonski diagran as shown in Fig. 1. The principle difference against fluorescence is the long triplet lifetime $\tau_{\text {p }}$ of the triplet state $T_{1}$ and as a result phosphorescence has usually only been observed in Pigid solutions at $77 \mathrm{~K}$.

For solutions of low concentration, where photo-chemical reactions such as dimerizations can be neglected, the phosphorescence quantum yield $\Phi_{p}$ is given by

$$
\Phi_{\mathbf{p}}=\theta_{\text {isc }} \mathbf{k}_{\mathbf{p}} /\left(\mathbf{k}_{\mathbf{p}}+\mathbf{k}_{\mathbf{n p}}+\sum_{\mathbf{Q} \mathbf{Q}}[Q]\right)=\theta_{\text {isc }} \mathbf{k}_{\mathbf{p}} \tau_{\mathbf{p}}
$$

where $\theta$ is the quantum yield of triplet formation. For other terms see Fig. 1. If the quenching process is diffusion controlled, $k_{Q}$ can be written as

$$
\mathbf{k}_{\mathbf{Q}}=8 \mathrm{RT} / 3000 \eta
$$

where $\eta$ denotes the viscosity of the liquid in poise (i.e., g.cm ${ }^{-1} . \mathrm{sec}^{-1}$ ) and $\mathrm{R}$ and $\mathrm{T}$ are the molar gas constant (erg. $\mathrm{deg}^{-1}$.mole $\mathrm{e}^{-1}$ ) and absolute temperature, respectively.

In rigid solutions at $77 \mathrm{~K}, \mathrm{k}_{\mathrm{Q}}$ becomes zero, so equation (1) is simplified to

$$
\Phi_{\mathbf{p}}=\theta_{\text {isc }} \mathbf{k}_{\mathrm{p}} /\left(\mathbf{k}_{\mathrm{p}}+\mathbf{k}_{\mathrm{np}}\right)
$$

hence quenching processes are suppressed and phosphorescence can be observed.

The requirement of rigid glassy solutions for the observation of phosphorescence appeared to be a serious hindrance for its use as a general analytical tool. Hence, a real breakthrough has been realized when it was shown that also at room temperature phosphorescence 


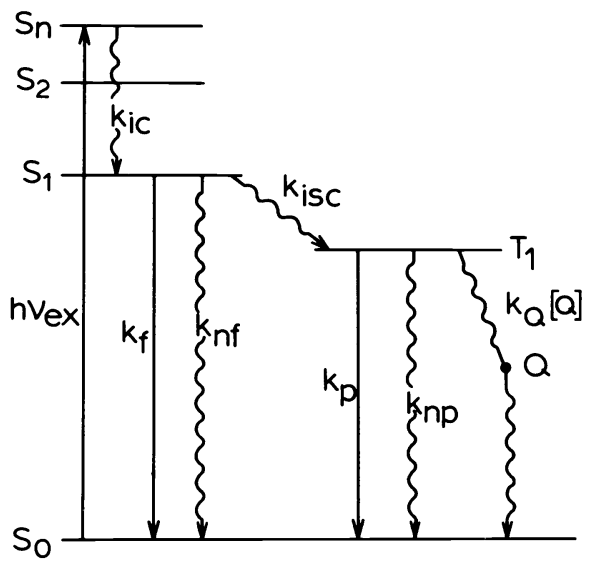

Fig. 1. Simplified diagram showing the kinetics of molecular excited states. $h \nu_{\text {ex }}$ indicates the frequency of the excitation light absorbed by the molecules; $k_{i c}$ is the rate constant of the internal conversion process, deactivating the higher excited singlet state $s_{n} ; k_{f}, k_{n f}$ and $k_{\text {isc }}$ are the rate constants of the fluorescence process, internal conversion process and intersystem crossing process resp., thus deactivating the $S_{1}$ state; $k_{p}$ and $k_{n p}$ are the rate constants of the phosphorescence process and Intersystem crossing process resp., which deactivate the $\mathrm{T}_{1}$ state; finally $\mathrm{k}_{Q}[\mathrm{Q}]$ is the effective rate constant of the bimolecular quenching reaction of this triplet state, given by $\mathrm{T}_{1}+$ $Q \stackrel{\mathbf{k}_{Q}}{\rightarrow} \mathrm{S}_{0}+\mathbf{Q}+$ heat.

can be observed for compounds being adsorbed to particular solid substrates like filter paper and silicagel. This type of phosphorescence, commonly denoted as RTP (room temperature phosphorescence) has found many applications in analytical chemistry, as has for instance been summarized by Lue Yen-Bower (2) and by Parker et al. (3). More recently it has been demonstrated that micellar solutions in the fluid state can emit strong phosphorescence at room temperature (Ref. 4) hence, the phosphorescing molecules are enclosed in micellar aggregates, formed by detergent monomers. The group of cline Love has extensively examined this phenomenon from an analytical point of view (Refs. 5-7)

Unfortunately, phosphorescence in common liquid solutions must be considered as quite exceptional. In 1958, Bäckström et al. (Ref. 8) have reported the phosphorescence of biacetyl in fluid benzene. In 1967, Almgren (Ref. 9) has shown that biacetyl phosphorescence is also observable in alkanes and even in water. In 1969 Parker and Joyce (Ref. 10) have published that phosphorescence signals can be obtained for several aryl ketones provided that extremely purified and thoroughly deoxygenated fluorocarbons are used as solvents. Nevertheless, in more common solvents, biacetyl and some related $\alpha$-diketones have been reported to be the only exceptions (Ref. 11). However, in 1978 Turro and coworkers (Ref. 4) demonstrated that also for brominated naphthalenes in acetonitrile strong phosphorescence signals can be recorded in a relatively simple way. Of course, phosphorescence of rigid glassy samples at $77 \mathrm{~K}$ is not simply applicable as a detection principle in dynamic flow systems. On the other hand, the application of phosphorescence at room temperature for compounds in the adsorbed state is somewhat more convenient. The principle of a detector using RTP has been described by Lloyd (12) who utilized a flow-cell packed with a mixture of crushed quartz and paper derived lint. Furthermore, the equipment developed by Miller (13), a rotating hollow drum wrapped around with filter paper or a TLC sheet, is a step in the direction of chromatographic detection. The same holds for the rotating mirror phosphorescope as has been described by Vo-Dinh et al. (14).

Room temperature phosphorescence in micellar solutions has the obvious advantage that it can be used as a detector in the fluid state. This principle has recently been applied by the group of Cline Love (Ref. 15) in two distinct ways: i) in a chromatographic system where the micellar solution was acting as the mobile phase and thus also influenced the separation and $i i)$ in a post-column addition mode where the micellar solution was added to the mobile phase after the separation step. With method i) sensitivities in the low nanogram range have been achieved. The sensitivities attainable with method ii) are lower because of the post-column mixing of eluent and micellar solution. However, method ii) has some advantages from the viewpoint of selectivity. 
Generally we must conclude, that even micellar media have their limitations when it comes to detector development. On the other hand, the encouraging results reported by Turro et al. (4) prompted us to take another look at the development of a phosphorescence detector which is in principle applicable under normal conditions for dynamic flow systems, in particular in liquid chromatography. Hence, we were only concerned with solvents, usually applied in LC, such as n-hexane and dichloromethane for normal phase chromatography or acetonitrile, methanol and water for reversed phase chromatography. Although the two most promising model compounds investigated, biacetyl (BIAC) and $1,4-$ dibromonaphthalene (Br $_{2} \mathrm{~N}^{\text {) }}$ exhibited excellent phosphorescence spectra under carefully deoxygenated conditions

(Ref. 16) it became clear rather quickly that direct RTPL as a detection mode would be of rather limited use. Hence, the two distinct detection methods based on RTPL described in this paper make use of the phosphorescence capacities of predominantly $\mathrm{BIAC}$ and $\mathrm{Br} 2 \mathrm{~N}$ in normal solvents.

In the LC experiments BIAC was added in small amounts to the eluent used for the separation (concentration on the order of $10^{-4} \mathrm{M}$ ). Basically, the two methods are i) measurement of the sensitized RTPL of BIAC after excitation of the analyte and subsequent energy transfer to BIAC and $i i)$ measurement of the partially quenched RTPL of BIAC, caused by the analyte.

\section{Sensitized RTPL}

The sensitized RTPL method can be readily outlined by means of the simplified diagram in Fig. 2. After excitation of the analyte (in the following denoted as the donor), different deactivation pathways are available. The molecules under consideration must have a high $k^{D}$ and thus a high triplet formation efficiency. In the absence of an acceptor in normal fluid systems the triplet is usually deactivated without phosphorescence, since $k_{n r}^{D}>$ $\mathbf{k}_{\mathbf{p}}^{\mathrm{D}}$. The crucial point of the sensitized RTPL method is that energy transfer from the donor

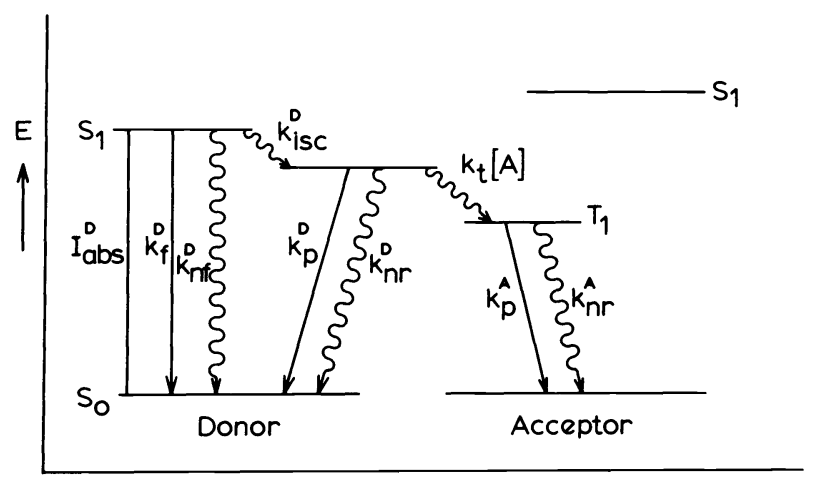
Fig. 2. Energy diagram for model system exhibiting sensitized phos-
phorescence. $I_{\text {abs }}^{D}=$ rate of light absorption by donor. $k_{f}^{D}$, $k_{n f}$ and $k_{i s c}^{D}$ phorescence. $I_{\text {abs }}=$ rate of light absorption by donor. $k_{f}$, $k_{n f}$ and $k_{\text {is }}$
are rate constants in $s^{-1}$ of intramolecular deactivation of donor via fluorescence, internal conversion and intersystem crossing, respectively. $\mathbf{k}_{p}^{\mathrm{D}}$ and $\mathbf{k}_{\mathrm{p}}^{\AA}$ are phosphorescence rate constants of donor and acceptor while $k_{p}$ are phosphorescence rate constants of donor and acceptor lecular non-radiative deactivation in $s^{-1} \cdot k_{t}[A]$ is apparent rate constant of energy transfer reaction in $s^{-1}$.

to the acceptor can compete successfully with the radiationless decay processes of the donor triplet and phosphorescence of the acceptor can be recorded. The intensity of the sensitized RTPL, denoted as I(sens), is given by (Ref. 17, 18)

$$
I \text { (sens) }=I_{\text {abs }}^{D} \cdot \theta_{i s c}^{D} \cdot \theta_{t}^{D A} \cdot \theta_{p}^{A}
$$

$I^{D}$ is the rate of light absorption by the donor; $\theta^{D}$ the efficiency of intersystem crossing and thus efficiency of triplet formation of the donor; $\theta$ the efficiency of energy transfer from donor to acceptor; and $\theta$ the phosphorescence efficiency of acceptor. $\theta_{t}^{D A}$ is the ratio of rate of energy transfer from donor to acceptor and overall decay rate of donor triplet:

$$
\theta_{t}^{D A}=k_{t}[A] /\left(\frac{1}{\tau_{0}^{D}}+k_{t}[A]\right)
$$


In equation (5) $k_{t}$ is the rate constant of the bimolecular energy transfer reaction $\left(\mathrm{M}^{-1}, \mathrm{~s}^{-1}\right)$ and $[A]$ the acceptor concentration. $\tau^{D}$ is the triplet lifetime of the donor in absence of the acceptor. The basic condition for the application of sensitized RTPL

$$
k_{t}[A]>\left(\tau_{0}^{D}\right)^{-1}
$$

can be met quite generally, provided that the triplet state energy of the acceptor is lower than that of the donor.

Direct excitation of the acceptor also leads to phosphorescence originating from the acceptor. Its intensity, denoted as $I($ dir), is given by

$$
I(\operatorname{dir})=\mathrm{I}_{\text {abs }}^{\mathrm{A}} \cdot \theta_{\text {isc }}^{\mathrm{A}} \cdot \theta_{\mathrm{p}}^{\mathrm{A}}
$$

In the sensitized RTPL detection method such direct phosphorescence is mainly responsible for the background signal, and hence an acceptor should be chosen which does not absorb in the excitation region of the donor. Biacetyl is an appropriate acceptor because its extinction coefficient is very low over a large range of wavelengths. The RTPL spectrum of biacetyl is given in Fig. 3 .

It is also important to notice that both $I$ (sens) and $I(d i r)$ are proportional to $\theta^{A}$, which strongly depends on the presence of oxygen and impurities in the solution. Therefbre the direct phosphorescence of the acceptor can be used to optimize the dynamic system. A typical schematic diagram for the apparatus used for measurements of sensitized RTPL signals in dynamic systems is given in Fig. 4 (see also Ref. 18). A $10^{-4}$ M BIAC solution in an acetonitrile/water mobile phase was used. A regular fluorescence detector and HPLC

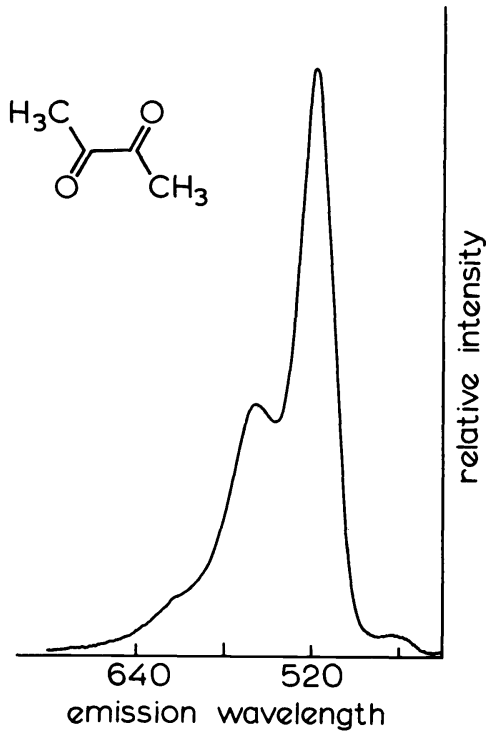

Fig. 3. Room temperature phosphorescence spectrum of biacetyl. The small peak at about $460 \mathrm{~nm}$ is due to fluorescence.

hardware (piston pump, column, loop injector) were adapted. The nitrogen gas used for deoxygenation of the eluent was purified on copper electrodes in the reduction column. Sample deoxygenation was also possible but in a chromatographic system the oxygen peak could be removed on the column. Connections were made in stainless-steel; teflon parts had to be avoided to prevent oxygen to leak into the system.

Extensive investigations for the application possibilities of sensitized RTPL were carried out with model systems such as chloro- and bromobiphenyls (PCBs, PBBs) and chloro- and bromonaphthalenes (PCNs, PBNs). Other successfully tested compounds were Cl-dibenzofuranes but dioxines were already too flexible with their two oxygen bridges between the aromatic ring systems. Rigidity and probably planarity of the molecular structure seems to be a necessary prerequisite for a good analyte as demonstrated by Weinberger (19), with the 


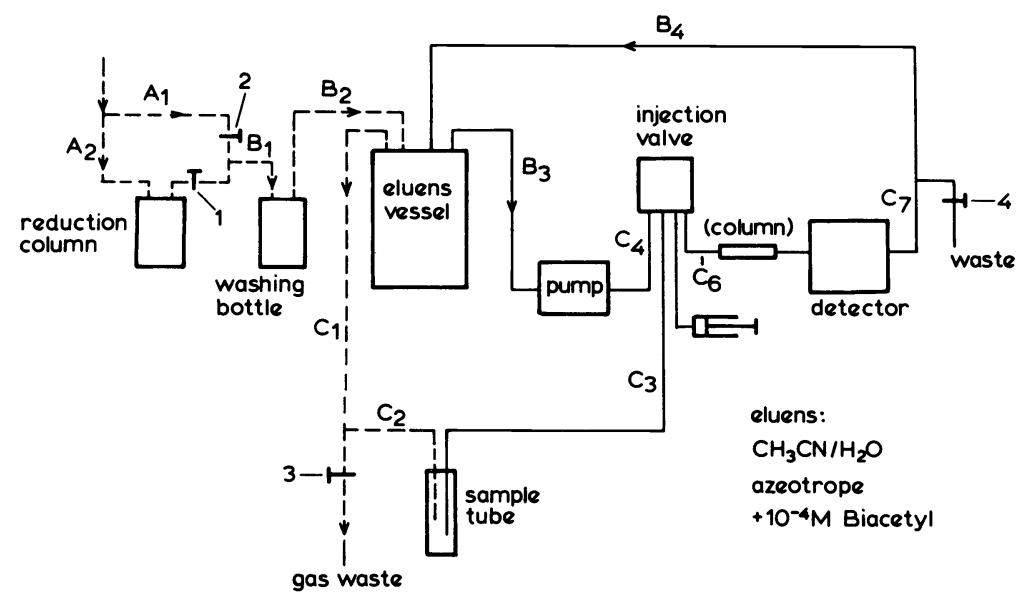

Fig. 4. Continuous flow system consisting of closed main circuit and injection system. Nitrogen capillaries depicted as broken lines; liquid capillaries as solid lines. A denotes brass capillaries $1 / 8$ " diameter, $B_{i}$ and $C_{i}$ indicate stainless steel capillaries $1 / 8$ " and $1 / 16$ " respectively. Valves 1 and 2 are type B-4HK (Nupro Comp., Cleveland, Ohio), valve 3 is a needle-valve (Hoekloos, Schiedam, The Netherlands) and valve 4 belonged to a model 332 Programmable Gradient System (Beckman/Altex, Mijdrecht, The Netherlands).

successful application of sensitized RTPL to cocaine and related molecules. Detection limits in the subnanogram range were obtained for most of these compounds. Although most experiments were carried out in water/acetonitrile media other solvents, conventionally used in HPLC such as methanol, dioxane, THF, hexane, etc. can be compatible with RTPL techniques.

Inspite of these positive results, the range of application of sensitized RTPL is still rather limited at the present stage. On the other hand, since the room temperature phosphorescence of biacetyl or 1,4-dibromonaphthalene is quite susceptible to oxygen quenching and other quenching processes, it seemed logical to also investigate the potential of phosphorescence quenching as a means of detecting certain groups of compounds.

\section{Quenched RTPL}

We have shown in the previous section that room temperature phosphorescence in liquid solutions (RTPL) can be successfully applied as a detection method in continuous flow and chromatographic systems if the triplet state of the analyte is higher in energy than the triplet state of biacetyl. An exothermic energy transfer is thus possible, which is generally diffusion controlled.

If the triplet energy of the analyte is equal to or lower than that of biacetyl, a reversed energy transfer reaction must be taken into account. This leads to both a decrease in the sensitized and the direct RTPL signal of biacetyl (Ref. 20). In the present section it is shown that measurement of such a partial quenching of the direct RTPL induced by the analyte can serve as a detection method in a continuous flow system. So in addition to sensitized RTPL detection, another phosphorescence detection method is available, denoted as quenched RTPL detection (see Table 1). Consequently, in general, analytes with triplet state energies too low to produce sensitized RTPL can be detected by quenched RTPL. It is emphasized that the application of quenched RTPL instead of sensitized RTPL requires only an adjustment of the excitation wavelength so that the same type of apparatus (see Fig. 4) can be used for both detection modes.

of course, in addition to triplet-triplet energy transfer, the analyte may also provide other deactivation pathways for the biacetyl triplet state, e.g., electron transfer.

The combination of UV detection, sensitized RTPL and quenched RTPL detection can give interesting results for the analyses of complex mixtures. This has been shown for some industrial mixtures of PCNs, which have been frequently used for impregnating purposes and for the PCB mixture Aroclor 1221 (Ref. 21).

The Halowaxes are a particularly interesting group of samples since they consist of complex mixtures of PCNs with different degrees of chlorination. Hence some PCNs have triplet 
TABLE 1. Reaction schemes of sensitized and quenched RTPL of biacetyl by triplet-triplet energy transfer

Sensitized RTPL Quenched RTPL

(1) Excitation of the analyte (A):

$$
\mathrm{A}+\mathrm{h} \nu_{\mathrm{ex}}^{\mathrm{A}} \longrightarrow{ }^{1} \mathrm{~A}^{*}
$$

(2) Intersystem crossing to the triplet state:

$$
{ }^{1} A^{*} \longrightarrow{ }^{3}{ }^{*}
$$

(3) Energy transfer to biacetyl (B):

$$
{ }^{3} A^{*}+B \stackrel{k_{t}}{\longrightarrow} A+{ }^{3} B^{*}
$$

(4) Phosphorescence of biacetyl:

$$
{ }^{3}{ }^{*} \longrightarrow \mathrm{B}+{ }_{h} \nu_{p}^{B}
$$

(1) Excitation of biacetyl (B):

$$
\mathrm{B}+\mathrm{h} \nu_{\mathrm{ex}}^{\mathrm{B}} \longrightarrow{ }^{1} \mathrm{~B}^{*}
$$

(2) Intersystem crossing to the triplet state:

$$
{ }^{1}{ }_{\mathrm{B}}{ }^{*}{ }^{3}{ }_{\mathrm{B}^{*}}
$$

(3) Quenching of biacetyl phosphorescence by the analyte:

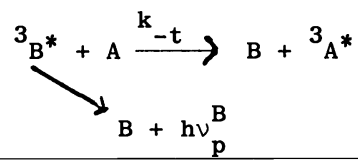

energy levels slightly above and others slightly below the energy level of biacetyl rendering as a rule PCNs with lower chlorination more susceptible to sensitized and PCNs with a higher degree of chlorination more suitable for quenched RTPL measurements.

As an example, the UV, sensitized RTPL, and quenched RTPL detected chromatograms of Halowax 1099 are depicted in Fig. 5 .

Peak 1 shows the presence of at least one of the three compounds $1,3-, 1,4-$ and $1,5-\mathrm{Cl} \mathbf{2}^{\mathrm{N}}$, sensitively detectable by sensitized, but not by quenched RTPL.

Peak 2 cannot be identified. It may be attributed to a dichloronaphthalene, i.e., 1,6-, 1,7- or 2,3-Cl $\mathrm{N}$. Its triplet state energy must be higher than that of biacetyl. Furthermore, comparison of the relative intensities of peaks 1 and 2 in Fig. 5A and 5B reveal that the unknown compound is a relatively poor sensitizer.

Peak 3 must be ascribed to $1,3,8-\mathrm{Cl}_{3} \mathrm{~N}$, which has both sensitizing and quenching properties (Ref. 20).

Peaks 5 and 11, only very weakly detected in the UV chromatogram (Fig. 5A), are well detectable in the sensitized one (Fig. 5B) since no influence of quenchers with the same retention time is encountered (Fig. 5C).

The quenching effect is pronounced for peaks 9 and 10, which are intense in Fig. 5A but hardly or not visible in Fig. 5B.

Peaks 6 and 7 deserve special attention. In the UV detected chromatogram they are not separated. However, in the sensitized RTPL detected chromatogram they are clearly separated into a negative and a positive peak. This can be simply explained on the basis of Fig. 5C which shows that peak 6 belongs to an efficient quencher, whereas peak 7 is not detectable by quenched RTPL. We presume that peak 6 corresponds to $1,4,5,8-C 1{ }^{N}$ ( $\operatorname{Ref}$. 7), which is a strong quencher because of its low lying triplet state (Ref. 8) and that peak 7 corresponds to $1,4,6-\mathrm{Cl}_{3} \mathrm{~N}$.

Peaks 12 and 13 consists of higher chlorinated PCNs which can have sensitizing as well as quenching properties.

Comparison of peak 14 in the three chromatograms reveals unambiguously, that it is composed of at least two components, one being only detectable by quenched RTPL and the other by both sensitized and quenched RTPL.

The application potential of quenched RTPL has been further explored (Ref. 22) since it seems to be a more widely applicable detection principle than the sensitized mode.

From a theoretical point of view the phenomenon can be treated as follows: the direct RTPL intensity of biacetyl, I(dir) is given by equation 7 . Changing the superscript $A$ into $B$ for biacetyl and in the absence of quencher $\left(\theta_{p}^{B}=k_{p}^{B} \cdot \tau_{o}^{B}\right)$,
equation 7 can be rewritten as:

$$
I(\operatorname{dir})=I_{\text {abs }}^{B} \cdot \theta_{i s c}^{B} \cdot \mathbf{k}_{p}^{B} \cdot \tau_{0}^{B}
$$

Here $k^{B}$ is the rate constant of the phosphorescence process (about $100 s^{-1}$ ) and $\tau_{0}^{B}$ the triplet lifetime of biacetyl. In deoxygenated and purified solvents $\tau_{0}^{B}$ ranges from $10^{-4}$ to $10^{-3} \mathrm{~s}$. 

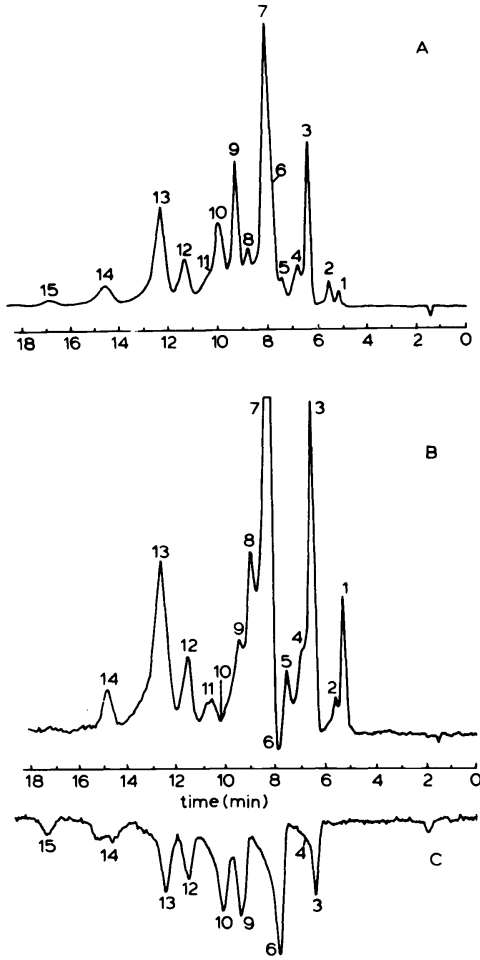

A

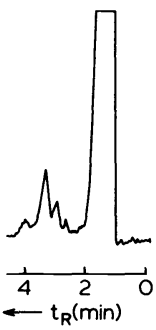

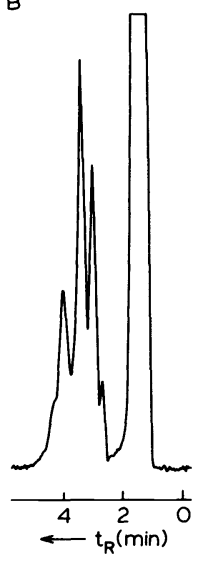

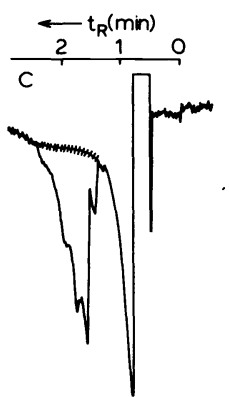

Fig. 6

Fig. 5. Chromatograms of Halowax 1099. A: UV detection; $\lambda_{\text {ex }}=233 \mathrm{~nm}$; 0.32 a.u.f.s. B: Sensitized RTPL detection; $\lambda_{\text {ex }}=300 \mathrm{~nm} ; \lambda_{\mathrm{em}}=520 \mathrm{~nm}$. C: Quenched RTPL detection; $\lambda_{e x}=415 \mathrm{~nm} ; \lambda_{\mathrm{em}}=520 \mathrm{~nm}$; injected amount of sample in all chromatograms: $1 \mu \mathrm{g}$. The identification of the peaks is given in the text. Separation conditions as in Fig. 6 .

Fig. 6. Chromatograns of $20 \mu 1$ urine sample containing Halowax 1001 . A : $100 \mathrm{ng}$ Halowax detected by quenched RTPL without signal inversion. The strongest peak corresponds to $\mathrm{O}_{2}$. B: $100 \mathrm{ng}$ Halowax detected by sensitized RTPL. $\lambda_{e x}=300 \mathrm{~nm}$. C: $10 \mathrm{ng}$ Halowax detected by sensitized RTPL. $\lambda_{\mathrm{ex}}=300 \mathrm{~nm}$. Chromatographic conditions: Eluent acetonitrile/ water $83.7 / 16.3 \mathrm{v} / \mathrm{v}$; RP-18 $5 \mu 11 \times 0.46 \mathrm{~cm}$ column. Flow $1 \mathrm{ml} / \mathrm{min}$.

If an amount of quencher is introduced, the triplet lifetime of biacetyl reduces to $\tau^{B}$ where:

$$
\left(\tau^{B}\right)^{-1}=\left(\tau_{0}^{B}\right)^{-1}+k_{Q}[Q]
$$

$k$ is the bimolecular rate constant of the quenching reaction and [Q] the concentration of quencher. As a result $I(d i r)$ decreases to $I^{\prime}($ dir) and one can derive a relationship similar to the well-known Stern-Volmer equation for fluorescence

$$
I(\operatorname{dir}) / I^{\prime}(\operatorname{dir})=1+k_{Q} \tau_{0}^{B}[Q]
$$

This equation demonstrates that there is a linear relationship between analyte concentra$t$ ion [Q] and 1/I'(dir), hence the use of a signal converter has been advocated by our group (Refs. 22, 23) to extend the linearity of our response curves. It also shows, why quenched phosphorescence is potentially such a powerful technique, since its sensitivity increases

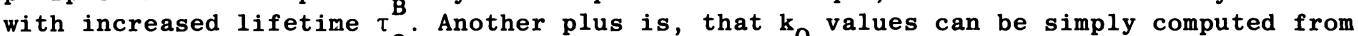
Stern-Volmer plots (Ref. 22) and from $k_{Q}$ values the ${ }^{Q_{\text {LODs }}}$ can be estimated which renders screening of potential analyte groups rather simple. Promising groups of compounds investigated (Ref. 22) with estimated LOD values in the order of $10^{-8}$ to $10^{-9} \mathrm{M}$, include anilines, other amines, heterocyclic aromatic nitrogen compounds, sulfur organics such as phenothiazines, thioureas etc. For all these compounds, beside possible energy back transfer mechanisms, electron transfer seems to be the major principle for the quenching to occur. For phenols quenched RTPL is also applied successfully but there this phenomenon cannot be explained by energy back transfer since their triplet energy level is higher than for biacetyl. Turro (kef. 24) ascribed it to a reversible hydrogen abstraction by the biacetyl triplet. An actual application of this principle to the chromatographic analysis of real samples (Ref. 23) is shown in Fig. 6 again for the detection of Halowax in human urine. Preconcentration and clean-up was done via a pre-column and direct on-line transfer of the sample to the analytical column. 
TABLE 2. $k$-values and order of estimated LODs for several inorganic ions in the azeotropic ${ }$ acetonitrile/water mixture.

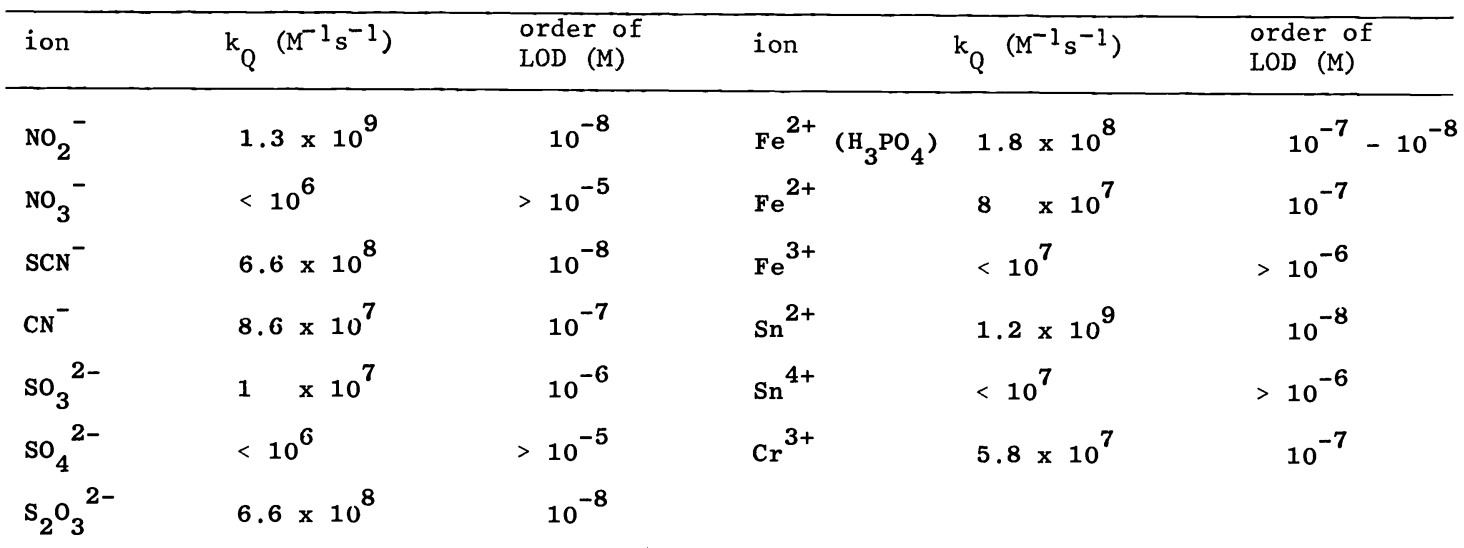

Detection in ion chromatography

Quenched RTPL has also shown considerable promise as a detector for selected inorganic ions in ion chromatography. A rather preliminary list of ions tested with experimentally determined $\mathrm{k}_{\mathrm{Q}^{-}}$and estimated LOD-values is given in Table 2 . The method has been applied in practice to develop a nethod for the analysis of nitrite in meat products and for the trace estimation of sulfite in wine (Ref. 25). This technique with its selectivity and sensitivity permits a reliable and reproducible analysis with a mininum of sample handling. The detection limits were one to two orders better than for other known methods.

One has to realize that with these few examples the surface of this field has only been scratched and numerous possibilities wait for exploration. Recent work with cis-platinum and other Pt(II) complexes used as cytostatics showed the great potential of quenched RTPL for these difficult to analyze compounds.

Future work on RTPL detection techniques will be in the direction of search for better sensitizers and primary phosphorophores (in place of biacetyl), improvement of electronics and design, i.e., by using pulse techniques and working with lifetime discrimination. Coupling RTPL with derivatization techniques, chemical excitation sources (chemiluminescence: and phosphorophores chemically bonded on solid substrates are but a few of the approaches which will be of interest in this domain.

\section{PHOTOSENSITIZED LUMINESCENCE}

The use of post-column reactions to generally enhance the luminescence and particularly fluorescence detection properties of various groups of analytes has been propagated for years (Ref. 26). A newer line in this direction is the development of procedures which do not require post-column addition of reagents and hence permit to eliminate reagent pumping and mixing problems. This can be achieved by adding reagents to the mobile phase and then, following separation, actuate the reaction catalytically, photochemically or by using a temperature increase. In some instances no reagent is needed or the reagent is made available as a solid substrate contained in a bed reactor.

In this section it is the photochemically generated or photosensitized reactions that are being discussed. A recent review (Ref. 27) is also dealing with this subject. We can say that photochemical reactions can be used as a special type of post-column derivatization, and improvements in both sensitivity and selectivity have been demonstrated. In the simplest case, photons are the only reagent added, and many examples of improved detection - particularly in the pharmaceutical area - have been demonstrated. For example, tamoxifen and clomiphene are stilbene derivatives (I), which upon irradiation with UV light are transformed into the corresponding phenanthrenes (II):

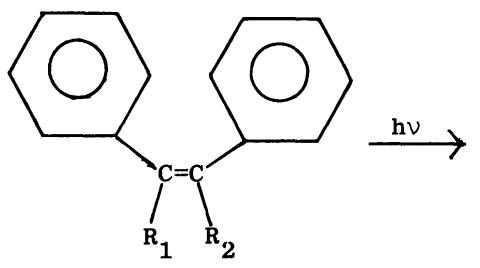

I

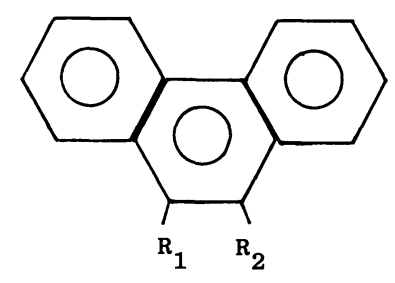

I I 
The increased rigidity and aromaticity of the phenanthrene molecule results in a much enhanced absorption coefficient and a higher fluorescence quantum yield. Selectivity for such analyses is generally high,hence biological tissues and fluids and complex environmental samples have been found to be particularly suitable for these techniques.

Another example of reagent free operation is the photochemical elimination of halogen substituents $(\mathrm{C} 1, \mathrm{Br})$ from an aromatic system and to detect the fluorescence of the product (anilines, phenoles) (Ref. 28). The inherent selectivity of this detection mode was again positive when analyzing phenoles or anilines in polluted water sources.

Generally we would say that post-column photochemical derivatization should be applicable to the determination of nearly all analytes for which batch photochemical methods have already been developed. In addition, the use of photochemical reagents greatly increases the applicability of the technique.

\section{Reactor design}

Aspects of reactor design and band broadening in photochemical detectors have been reviewed earlier (Refs. 26, 27). A typical reactor unit is shown in Fig. 7. A Xe-Hg arc lamp is most often used since its high intensity over the entire UV-visible region makes it a generally useful excitation source. Optical filters can, in principle, be used to increase the selectivity of the photochemical reaction, although this has yet to be demonstrated.

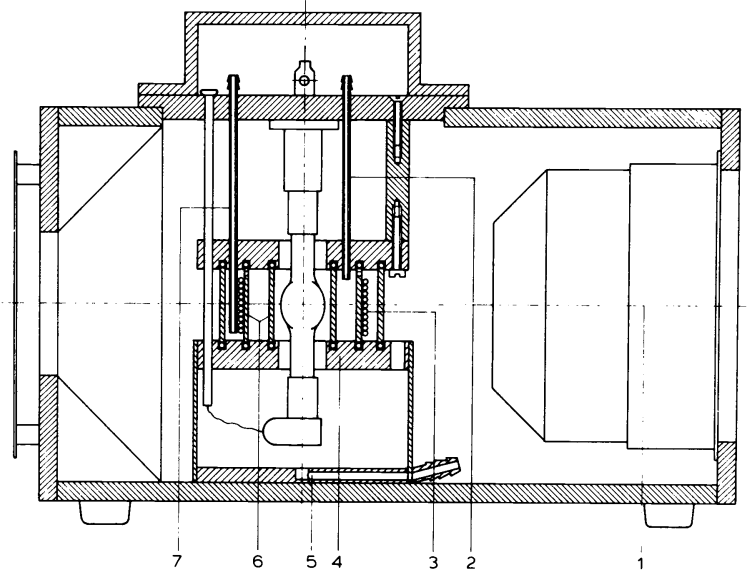

Fig. 7. Photochemical reactor. 1, fan; 2, outlet for circulating 1iquid; 3, capillary; 4, filter compartment; 5, additional cooling via pressurized air; 6, quartz cylinders; 7, liquid inlet. The light source is a $200 \mathrm{~W} \mathrm{Hg}-\mathrm{Xe}$ lamp operated at a current of 10A. The PTFE reaction coil is cooled by a continuous flow of water through the jackets by two quartz and one pyrex cylinder.

A disadvantage of arc lanps is that they produce a great deal of heat and infrared radiation, and thus cooling of both the lamp and reaction coil are required. High temperatures within the reaction coil can result in undesirable side reactions, including polymerization. Furthermore, temperature fluctuations adversely affect the reproducibility. The reactor in Fig. 7 makes use of a polytetrafluoroethylene (PTFE) reaction coil. PTFE has been found to transmit light by an internal reflection mechanism in which light passes through the pores of the polymer. Some reactor designs have utilized quartz capillaries. However, these are both expensive and fragile. They are not readily available in different geometries (coil and helix diameter, length) and tight connections are difficult to make. The surprisingly high transmission of light in the 200-300 nm spectral region by PTFE makes this material nearly ideal for photochemical reactors, especially since the tubing is inexpensive and readily available in a range of internal diameters. Also, it has been shown that the use of PTFE coils results in chromatographic peaks having better symmetry and less tailing than for quartz capillaries (Ref. 29). The only disadvantage of PTFE reaction coils is that the material is permeable to oxygen, so that the sensitivity of detection may be reduced for certain analytes if the reaction coil is exposed to air. In the reactor design of Fig. 7, the PTFE coil is immersed in a circulating water bath which serves both to regulate the temperature and to exclude oxygen. Also, metal salts may be added to the water bath to serve as an optical filter.

\section{Applications}

The post-column photochemical derivatization has been applied most widely for various pharmaceuticals which have been listed earlier (Ref. 27). Only one example, Vitamin $K_{1}$ (Ref. 30), will be discussed here since it illustrates a number of important considera- 
tions in photochemical reaction detection. To begin with, the photochemistry of complex molecules is seldom sinple. This is illustrated by Fig. 8, which summarized the literature on the photochemistry of Vitamin $k_{1}$ solutions. The reaction products are highly dependent on solvent composition, the presence or absence of oxygen, pH and irradiation time.

The Vitamin $\mathrm{K}$ homologues do not exhibit native fluorescence; however, in batch photolysis experiments it was found that at least three fluorescent products were formed. It was also observed that in the presence of small concentrations of ascorbic acid the only isolable fluorescent product is the hydroquinone $\mathrm{KH}_{2}$. The ascorbic acid acts as a chemical reductant and protects the $\mathrm{KH}_{2}$ from further oxidative decomposition. This is thus a typical example how the addition of (low concentrations) reagent can render the photochemical reaction more controllable and better defined.

Because of hydroquinone is highly fluorescent and can be reproducibly formed in good yield, its optimum excitation and emission wavelengths were chosen for the analytical procedure. Purging the solvent system with nitrogen improved the sensitivity by up to $50 \%$, as would be expected, considering the various reactions with $\mathrm{O}_{2}$ shown in $\mathrm{Fig}$. 8 .

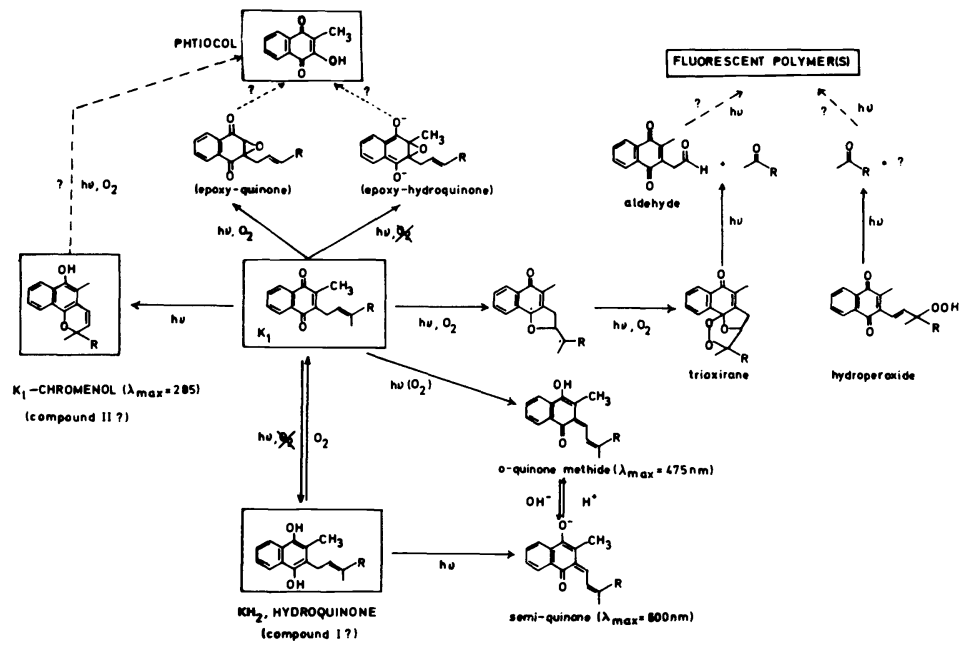

Fig. 8. Proposed photochemical reactions of Vitamin $K_{1}$.
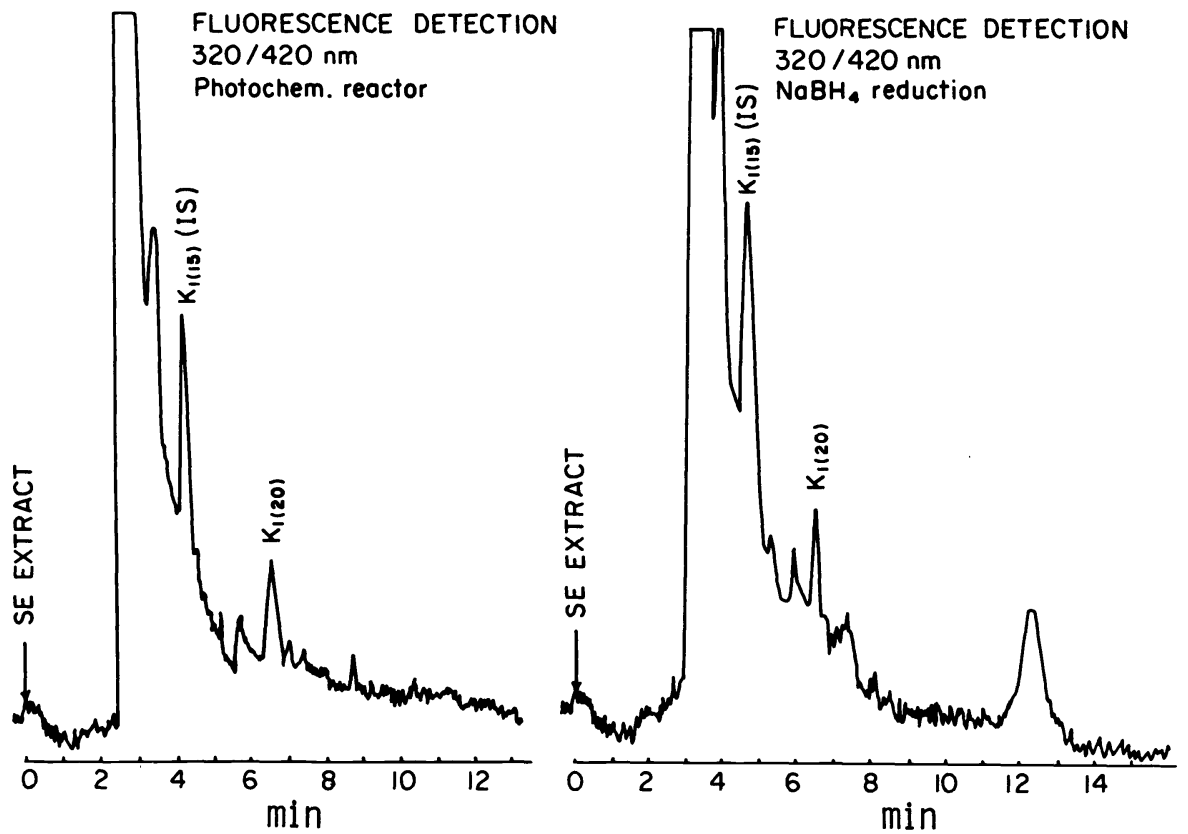

Fig. 9. Column eluate of a serum extract monitored with photochemical reaction detection; and with fluorescence detection after $\mathrm{NaBH}_{4}$ reduction. $\mathrm{K}_{1(20)}$ : native $\mathrm{K}_{1}$ (phylloquinone); $\mathrm{K}_{1(15)}$ : a synthetic homolog of $K_{1}$ with $^{1} \mathrm{a}^{15} \mathrm{C}$ side chain, used as internat (15) $)^{1}$ andard (I.S.). Separation conditions: $15 \mathrm{~cm} \times 3.2 \mathrm{~mm}$ I.D., RP18 $5 \mu$ column; eluent : $\mathrm{CH}_{3} \mathrm{OH} / \mathrm{H}_{2} \mathrm{O}$ (98:2); flow-rate: $1 \mathrm{ml} / \mathrm{min}$. 
Figure 9 shows a chromatogram for Vitamin $\mathrm{K}_{1}$ in a serum extract detected via photochemical reaction and also by post-column addition of a reducing reagent. Apart from the added convenience of photochemical reduction we also observe less background (slightly higher selectivity) for the photochemical technique. Generally we can say that the post-column reaction permits direct analysis of the Vitamin $K_{1}$ in a simple serum extract, which if detected by UV would require extensive clean-up procedures.

The application just discussed requires, like most others (Refs. 26, 27), that the analyte absorbs light to initiate the photochemical reaction. A photochemical reduction similar to that described above for Vitamin $K_{1}$ has recently been used to detect a variety of compounds that have only very weak chromophores in the UV-visible region, or which have none at all (Refs. 31, 32). In this detection scheme the compound anthraquinone-2,6-disulfonate $(Q)$ is photoreduced to the hydroquinone in the presence of analytes having $\mathrm{C}-\mathrm{H}$ bond strengths less than about $94 \mathrm{kcal} / \mathrm{mol}$. The mechanism of photoreduction of isopropanol is shown in Fig. 10. For this reaction schene the (Q) reagent absorbs light and undergoes rapid intersystem crossing to the triplet state. This triplet excited species can abstract the $\alpha$-hydrogen to produce a semiquinone radical $\left(\mathrm{QH}^{\circ}\right)$ and the $\left(\mathrm{CH}_{3}\right)_{2} \mathrm{COH}$. radical. Subsequent reactions lead to the fully reduced and highly fluorescent hydroquinone, $Q H_{2}$. Using a fluorescent black light as a source and a reaction time of $26 \mathrm{~s}$, detection limits in the range 5-30 ng were achieved for a variety of aliphatic alcohols, aldehydes, ethers and saccharides. As it stands, this post-column photochemical reaction technique is more than an order of magnitude more sensitive than a conventional refractive index detector and provides the benefit of considerable selectivity. In an alternate detection scheme for such compounds, $Q$ acts as a sensitizer in a photo-oxygenation (Ref. 32). The reaction mechanism for ethanol is shown in Fig. 11. Rather than exclude oxygen,

$$
\begin{aligned}
& Q+h \nu \rightarrow Q^{*} \text { (singlet, } \mathrm{S} \text { ) } \\
& \mathrm{Q}^{*}(\mathrm{~S}) \longrightarrow \mathrm{Q}^{*} \text { (triplet, } \mathrm{T} \text { ) } \\
& \mathrm{Q}^{*}(\mathrm{~T})+\mathrm{CH}_{3} \mathrm{CH}_{2} \mathrm{OH} \longrightarrow \mathrm{QH} \cdot+\mathrm{CH}_{3} \mathrm{CHOH} \\
& \mathrm{CH}_{3} \mathrm{CHOH}+\mathrm{Q} \longrightarrow \mathrm{QH} \cdot+\mathrm{CH}_{3} \mathrm{CHO} \\
& 2 \mathrm{QH} \cdot \rightarrow \mathrm{Q}+\mathrm{QH}_{2}
\end{aligned}
$$

Fig. 10. Mechanisn for the photoreduction of anthraquinone by isopropanol. $Q$ is anthraquinone; $\mathrm{QH}$. is the semiquinone radical; $\mathrm{QH}_{2}$ is $9,10-\mathrm{di}-$ hydroxyanthracene.

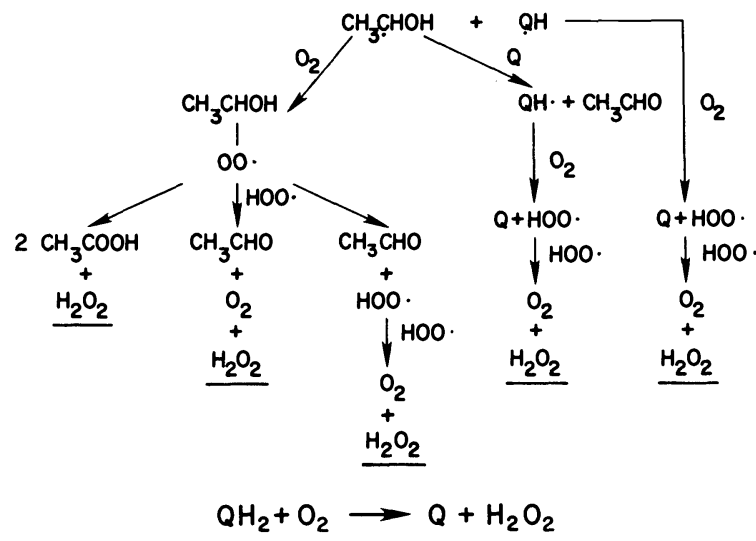

Fig. 11. Mechanism for the sensitized photo-oxygenation of ethanol in neutral aqueous solution. $Q$ is a sensitizer.

as is necessary for the photoreduction, the HPLC mobile phase is saturated with oxygen by continuously bubbling $O_{2}$ through the solvent reservoir. Hydrogen peroxide is a major product of the reaction and may be detected by a suitable chemiluminescent reaction (Ref. 32) or electrochemically. An important difference between the photo-oxygenation (Fig. 11) and photoreduction (Fig. 10) mechanisms is that in the photo-oxygenation the $Q$ is not consumed, but rather acts as a catalyst. This suggests the potential as a kinetic method in which $Q$ functions as the analyte which sensitizes the photo-oxygenation of an alcohol. Using an alcohol/water mobile phase saturated with $\mathrm{O}_{2}$, it should be possible to detect very weak sensitizers at low concentrations, since the reaction of the triplet-excited analyte with the alcohol would be quite favourable in comparison to the $T_{1} \rightarrow S_{0}$ deactivation and because long reaction times in the photochemical reactor would allow very high quantum yields of $\mathrm{H}_{2} \mathrm{O}_{2}$. 
With these finally discussed photosensitation techniques, the use of photochemical reaction detectors can take place with a much wider range of compounds and related reaction mechanisms are better known and defined. The potential of photochemical reaction detectors is thus greatly enhanced.

\section{CONCLUSIONS AND FUTURE ASPECTS}

We could argue that the common theme for the various luminescence techniques discussed in this paper is based on the use of intermolecular energy transfer phenomena for detection in liquid streams. Obviously the present treatment is by no means comprehensive, it gives, in fact, just a few glimpses into a still totally open and very little explored field. In the last section, cheniluminescence was mentioned for the detection of $\mathrm{H}_{2} \mathrm{O}_{2}$. This is another area where such transfer mechanisms play a key role. The idea of chemical excitation sources replacing electronic ones, is very attractive from a selectivity, sensitivity as well as cost factor point of view. Much attention will have to be paid in future research to this aspect. Another argument is, that chemiluminescence as well as the other luminescence modes discussed, are quite suitable for miniaturization, hence detectors can be developed on this basis, which are compatible with the current developments in micro LC both in terms of low disperion and response time characteristics.

\section{REFERENCES}

1. R.W. Frei and J.F. Lawrence, Chemical Derivatization in Analytical Chemistry, 1 , Plenum Press, New York (1982).

2. E. Lue Yen-Bower, J.L. Ward, G. Walden and J.D. Winefordner, Talanta 27, 380 (1980).

3. R.T. Parker, R.S. Freedlander and R.B. Dunlop, Anal. Chim. Acta 119,189 , and $120,1 \quad(1980)$.

4. N.J. Turro, L. Kou Chiang, C. Ming-Fea and P. Lee, Photochem. and Photobiol. 27, $523(1978)$.

5. L.J. Cline Love, M. Skrilec and J.G. Habarta, Anal. Chem. 52, 754

6. M. Skrilec and L.J. Cline Love, Anal. Chem. 52, 1559 (1980).

7. L.J. Cline Love and M. Skrilec, $\overline{\text { Anal. Chem }} . \overline{53}, 1872 \quad$ (1981).

8. H.L.J. Bäckström and K. Sandros, Acta Chem. Scand. 12,823

9. M. Almgren, Photochem. and Photobiol. 6, 829 (1967)

10. C.A.Parker and T.A. Joyce, Trans. Faraday Soc. 65, 2823

11. H.H. Richtol and F.H. Klappreier, J. Chem. Phys. 44, 1519 (1958).

(1980)

12. J.B.F. Lloyd, Analyst 103,775 (1978)

13. J.N. Miller, Trends in Anal. Chem. 1, 31 (1981).

14. T. Vo-Dinh, G.L. Walden and J.D. Winefordner, Anal. Chem. 49, 1126

(1969) .

(1966).

15. R. Weinberger, P. Yarmchuk and L.J. Cline Love, Anal. Chem. 54, 1552

16. J.J. Donkerbroek, J.J. Elzas, C. Gooijer, R.W. Frei and N.H. Velthorst, Talanta 28, 717-723 (1981).

17. J.J. Donkerbroek, C. Gooijer, N.H. Velthorst and R.W. Frei, Anal. Chem. 54, 891-895 (1982)

18. J.J. Donkerbroek, N.J.R. van Eikema Hommes, C. Gooijer, N.H. Velthorst and R.W. Frei, Chromatographia 15, 218-222 (1982).

19. R. Weinberger, Kratos Inc. New Jersey, personal communication.

20. J.J. Donkerbroek, A.C. Veltkamp, A.J.J. Praat, C. Gooijer, R.W. Frei and N.H. Velthorst, Applied Spectr. 37, 188-192 (1983).

21. J.J. Donkerbroek, N.J.R. van Eikema Hommes, C. Gooijer, N.H. Velthorst and R.W. Frei, J. Chromatogr. 255, 581-590 (1983).

22. J.J. Donkerbroek, A.C. Veltkamp, C. Gooijer, N.H. Velthorst and R.W. Frei, Anal. Chem. 55, 1886-1893 (1983).

23. J.J. Donkerbroek, C. Gooijer, N.H. Velthorst and R.W. Frei, Inter. J. Environ. Anal. Chem. 15, 281-301 (1983).

24. N.J. Turro and R. Engel, J. An. Chem. Soc. 91, 7113 (1969).

25. C. Gooijer, P.R. Markies, J.J. Donkerbroek, N.H. Velthorst and R.W. Frei, J. Chromatogr. 289, 347-354 (1984).

26. R.W. Frei, "Reaction detectors in liquid chromatography", in chemical reactions in analytical chemistry, Volume I (Eds. R.W. Frei and J.F. Lawrence), Plenum Press, New York (1982).

27. J.W. Birks and R.W. Frei, Trends in Anal. Chem. 1, 361-367 (1982).

28. C.E. Werkhoven-Goewie, W.M. Boon, A.J.J.Praat, R.W. Frei, U.A.Th. Brinkman and C.J. Little, Chronatographia 16, 53-59 (1983).

29. A.H.M.T. Scholten, P.L.M. Welling, U.A.Th. Brinkman and R.W. Frei, J. Chromatogr. $199,239-248$ (1980).

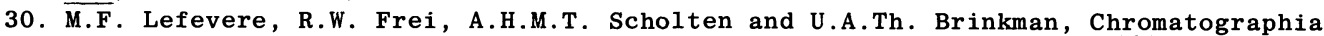
$15,459-467$ (1982).

31. M.S. Gandelman and J.W. Birks, Anal. Chem. 54, 2131 (1982).

32. M.S. Gandelman, J.W. Birks, U.A.Th. Brinkman and R.W. Frei, J. Chromatogr. 282, 193-209 (1983) 